\title{
(di)lepton physics with ALICE
}

\author{
P. Crochet $^{\mathrm{a}, *}$ for the ALICE Collaboration

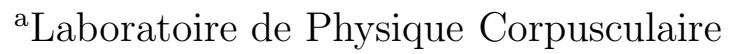 \\ CNRS/IN2P3 and Université Blaise Pascal \\ F-63000 Clermont-Ferrand, France
}

Physics perspectives with (di)lepton measurements with the ALICE detector at the LHC are reviewed. Special emphasis is placed on heavy flavor physics.

\section{1. (di)leptons and heavy flavors: what is different at the LHC}

One of the most exciting aspects of heavy ion collisions at the LHC is the abundant production rate of heavy flavors which can be used, for the first time, as high statistics probes of the medium [1, 2]. This allows to use a large variety of new observables. The magnitude of most of the in-medium effects is expected to be dramatically enhanced. Some of these aspects are discussed hereafter.

- Large primary production cross-sections: The number of $c \bar{c}(b \bar{b})$ pairs produced in central $A A$ collisions at the LHC is expected to be 10 (100) times larger than at RHIC. Therefore, at the LHC both charmonia and bottomonia can be used, thus providing powerful probes for Quark Gluon Plasma (QGP) studies. In fact, since the $\Upsilon(1 S)$ state is expected to only dissolve significantly above the critical temperature [ [3, 4], which might only be reachable at the LHC, the spectroscopy of the $\Upsilon$ family should reveal unique characteristics of the QGP [ 5];

- Large resonance dissociation rate: In addition to nuclear absorption, comoving hadrons and color screening, quarkonia can be significantly destroyed by gluon bombardment [ 6]. This mechanism which results from the presence of quasifree gluons starts being effective for temperatures above the critical temperature but not necessarily above the resonance dissociation temperature by color screening. It is expected to be relatively important at the LHC. Indeed, recent estimates [ [2] of the dissociation cross-sections show that none of the prompt $J / \psi$ would survive the deconfined phase at the LHC and that about $80 \%$ of the $\Upsilon$ would be destroyed;

- Large charmonium secondary production: Besides indirect charmonia production from $b$-hadron decay [7] (see below) an important yield of secondary charmonia is expected from $D \bar{D}$ annihilation [ 8], statistical hadronization [9] and kinetic recombination [10]. The two last processes explicitly assume the formation of a deconfined medium. The underlying picture is that charmonium resonances form

\footnotetext{
*crochet@clermont.in2p3.fr
} 
by coalescence of free $c$ and $\bar{c}$ in the QGP [10] or at the hadronization stage [9]. According to these models, the signature of the QGP should lead to an increase of the $J / \psi$ yield versus centrality, proportional to $\mathrm{N}_{c \bar{c}}^{2}$, instead of a suppression ${ }^{2}$;

- Complex structure of (di)lepton spectra: With a low $p_{\mathrm{t}}$ threshold of about $2 \mathrm{GeV} / \mathrm{c}$ on the decay leptons, unlike-sign dileptons from bottom decay dominate the dilepton correlated component all over the mass range. Whereas in the high invariant mass region each lepton comes from the direct decay of a $B$ meson, in the low invariant mass region both leptons come from the decay of a single $B$ meson via a $D$ meson. Next-to-leading order processes such as gluon splitting also populate significantly the low mass dilepton spectrum due to their particular kinematics [ 12. Then, a sizeable yield of like-sign correlated dileptons from bottom decay is present. This contribution arises from the peculiar decay chain of $b$ hadrons and from $B$-meson oscillations. The single lepton spectra are also subject to significant novelties. The most striking one is the emergence of the $W^{ \pm}$bosons as a bump located at around $30 \mathrm{GeV} / \mathrm{c}$ in the single lepton $p_{\mathrm{t}}$ distributions [13].

\section{Selected physics channels}

ALICE (A Large Ion Collider Experiment) is the LHC experiment dedicated to the study of nucleus-nucleus collisions. The detector consists of a central barrel $(|\eta|<0.9)$, a forward muon spectrometer $(2.5<\eta<4)$ and several forward/backward and central small acceptance detectors [1, 14. (di)leptons will be measured in ALICE through the electron channel in the central region and through the muon channel in the forward region. Selected physics channels are presented below.

- $\Upsilon^{\prime} / \Upsilon$ ratio versus $p_{\mathrm{t}}$ : The $p_{\mathrm{t}}$ suppression pattern of a resonance is a consequence of the competition between the resonance formation time and the QGP temperature, lifetime and spatial extent [15]. Quarkonium suppression is expected also as the result of nuclear effects like shadowing and absorption. In order to isolate pure QGP effects, it has been proposed to study the $p_{\mathrm{t}}$ dependence of quarkonium ratios instead of single quarkonium $p_{\mathrm{t}}$ distributions. By doing so, nuclear effects cancel out, at least in the $p_{\mathrm{t}}$ variation of the ratio. Following the arguments of [ 5], the capabilities of the ALICE muon spectrometer to measure the $p_{\mathrm{t}}$ dependence of the $\Upsilon^{\prime} / \Upsilon$ ratio in central $(10 \%) \mathrm{Pb}-\mathrm{Pb}$ collisions have been investigated [ 16]. Two different QGP models with different system sizes were considered. The results of the simulations show that, with the statistics collected in one month of data taking, the measured $\Upsilon^{\prime} / \Upsilon$ ratio exhibits a strong sensitivity to the characteristics of the QGP;

- Secondary $J / \psi$ from $b$-hadron decay: As stated above, a large fraction of $J / \psi$ arises from $b$-hadron decay ${ }^{3}$. These secondary $J / \psi$, which are not QGP suppressed,

\footnotetext{
${ }^{2}$ Note that, due to the large number of $c \bar{c}$ pairs produced in central heavy ion collisions at LHC, these models predict a spectacular enhancement of the $J / \psi$ yield, up to a factor 100 in central collisions, relative to the primary production yield [ 2, 11].

${ }^{3}$ In central $(5 \%) \mathrm{Pb}+\mathrm{Pb}$ collisions at $\sqrt{s}=5.5 \mathrm{TeV}, \mathrm{N}(b \bar{b} \rightarrow J / \psi) / \mathrm{N}($ direct $J / \psi) \sim 20 \%$ in $4 \pi$ (with shadowing and feed-down but without nuclear absorption) [2].
} 
must be subtracted from the measured $J / \psi$ yield prior to $J / \psi$ suppression studies. They can be identified by exploiting the large lifetime of $b$ hadrons which results in a finite impact parameter for the decay leptons of secondary $J / \psi$. Simulations have shown that such measurements can successfully be performed with dielectrons measured in the central part of ALICE thanks to the excellent spatial resolution of the Inner Tracking System [17];

- Open heavy flavors: The open heavy flavor cross-section can be measured by means of several channels: low-mass and high-mass unlike-sign dileptons [18], single lepton $p_{\mathrm{t}}$ distributions [18, 19, like-sign dileptons [20], single leptons with displaced vertices [17, 19], secondary $J / \psi$ from $b$-hadron decay [17] and electron-muon coincidences [17]. Recently, a measurement of the differential inclusive $b$-hadron cross-section has been investigated in the electron channel [19] with a technique developed in $p \bar{p}$ collisions [ 22 and adapted to heavy ion collisions in the ALICEmuon channel [18]. The results presented in Figure1] (left) show that the $b$-hadron cross-section can be reconstructed up to $p_{\mathrm{t}}^{b}$ hadron $=30 \mathrm{GeV} / \mathrm{c}$. Sensitivity to the $b$-quark energy loss is evidenced such that the nuclear modification factors, which can be simultaneously measured for light hadrons, for $D^{0}$ [23] and for $b$ hadrons should provide a set of powerful tools to investigate the mass dependence of the energy loss;
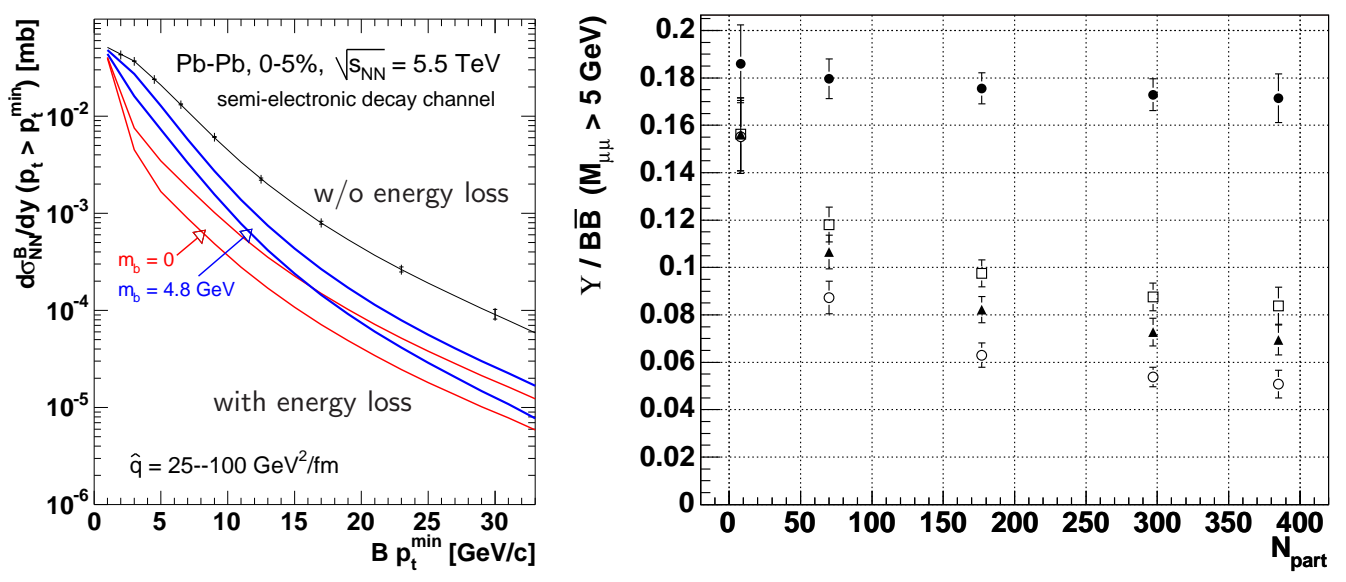

Figure 1. Left: differential inclusive $b$-hadron cross-section reconstructed from single electrons with displaced vertices in central $(5 \%) \mathrm{Pb}+\mathrm{Pb}$ collisions [19, 21]. The results are shown without and with $b$-quark energy loss according to [24]. Right: centrality dependence of the $\Upsilon / b \bar{b}$ ratio in $\mathrm{Pb}+\mathrm{Pb}$ collisions in the muon channel. The ratio is shown without (dots) and with (squares) $\Upsilon$ nuclear absorption as well as with nuclear absorption and melting by color screening (triangles and circles) with dissociation temperatures taken from [4]. Taken from [21, 25].

- Centrality dependence of the $\Upsilon / b \bar{b}$ ratio: If the $b$-quark energy loss turns out to be negligible, the $b$-hadron cross-section can be used as a normalisation for $\Upsilon$ suppression studies. This normalisation is the most natural normalisation because of the similar production processes for open and hidden heavy flavors. Figure 1 (right) shows the measurement that can be achieved in one month of $\mathrm{Pb}$ beams. 
The triangles and circles illustrate the typical sensitivity of the ratio to $\Upsilon$ melting by color screening. Note that the statistical uncertainty of the ratio is dominated by the statistics of the probe (i.e. the number of $\Upsilon_{\mathrm{s}}$ ) and not by the statistics of the reference. Indeed, the number of correlated unlike-sign muon pairs from bottom decay in the mass range $\mathrm{M}_{\mu \mu}>5 \mathrm{GeV} / \mathrm{c}^{2}$ is larger than that of the $\Upsilon$ by a factor 5 .

\section{Summary}

(di)lepton measurements with the ALICE detector at the LHC will bring an unprecedentedly rich physics program in the heavy flavor sector of heavy ion collisions. In addition to the channels discussed here, further exciting possibilities should be opened with, for example, quarkonia polarization and dilepton correlations.

\section{Acknowledgments}

Part of this work was supported by the EU Integrated Infrastructure Initiative HadronPhysics Project under contract number RII3-CT-2004-506078.

\section{REFERENCES}

1. ALICE Collaboration, J. Phys. G 30 (2004) 1517.

2. M. Bedjidian et al., arXiv:hep-ph/0311048.

3. S. Digal et al., Phys. Rev. D 64 (2001) 094015.

4. C. Y. Wong, arXiv:hep-ph/0408020; W. M. Alberico et al., arXiv:hep-ph/0507084

5. J. F. Gunion and R. Vogt, Nucl. Phys. B 492 (1997) 301.

6. X. M. Xu et al., Phys. Rev. C 53 (1996) 3051.

7. S. Eidelman et al., Phys. Lett. B 592 (2004) 1.

8. P. Braun-Munzinger and K. Redlich, Eur. Phys. J. C 16 (2000) 519 and ref. therein.

9. P. Braun-Munzinger and J. Stachel, Phys. Lett. B 490 (2000) 196.

10. R. L. Thews, M. Schroedter and J. Rafelski, Phys. Rev. C 63 (2001) 054905.

11. A. Andronic et al., Phys. Lett. B 571 (2003) 36.

12. E. Norrbin and T. Sjostrand, Eur. Phys. J. C 17 (2000) 137.

13. Z. Conesa del Valle for the ALICE Collaboration, http://www.to.infn.it/qgp/welcome05.html.

14. http://aliceinfo.cern.ch/index.html; H.-A .Gustafsson, these proceedings.

15. F. Karsch and H. Satz, Z. Phys. C 51 (1991) 209 and ref. therein.

16. E. Dumonteil and P. Crochet, ALICE-INT-2005-002 (2005).

17. ALICE Collaboration, CERN/LHCC 2001-021 (2001).

18. R. Guernane et al., ALICE-INT-2005-018 (2005).

19. A. Dainese for the ALICE Collaboration, http://hcp-2005 . web .cern.ch/HCP-2005/.

20. P. Crochet and P. Braun-Munzinger, Nucl. Instrum. Meth. A 484 (2002) 564.

21. ALICE Collaboration, Physics performance report Vol. 2, in preparation.

22. C. Albajar et al. [UA1 Collaboration], Phys. Lett. B 213 (1988) 405.

23. A. Dainese [ALICE Collaboration], Eur. Phys. J. C 33 (2004) 495.

24. N. Armesto et al., Phys. Rev. D 71 (2005) 054027.

25. S. Grigoryan and R. Guernane, private communication (2005). 